\title{
Towards a Culturally Aware Design for Social Software
}

\author{
Roberto Pereira \\ University of Campinas
}

\author{
Leonardo Cunha de Miranda \\ Federal University of Rio \\ Grande do Norte
}

\author{
M. Cecília C. Baranauskas \\ University of Campinas
}

\author{
Lara S. Godoy Piccolo \\ $C P q D$ and University of \\ Campinas
}

\author{
Leonelo Dell Anhol Almeida \\ University of Campinas
}

\author{
Julio Cesar dos Reis \\ CTI and University of \\ Campinas
}

\begin{abstract}
Cultural aspects, such as values, beliefs and behavioral patterns influence the way technology is understood and used, and the impact it may cause on the environment and on people. Social software is a growing reality worldwide, while the interaction design of this kind of systems is still a challenging task due to the lack of artifacts, methods, tools and even theories for supporting designers in dealing with subjective and contextualized issues, such as the cultural ones. In this paper, we present a culturally aware artifact named VF4SS that can support designers in a requirements clarification activity. We demonstrate how it can be used for dealing with cultural issues in an explicit way, suggesting a practical guide to support designers in this task. We draw on Organizational Semiotics and on building blocks of culture to ground our discussions and situate them in a practical context related to the development of social software for professionals of the Special Education field.
\end{abstract}

\section{Introduction}

The so-called social software that emerged and became popular after the Web 2.0 advent is frequently cited as determinant of transformations that are changing the way people relate to technologies [1]. Systems such as Facebook and Youtube are examples of how technology has left the workplace context to pervade every aspect of people's personal and social life. This kind of system is used not only at home but also in schools, workplaces, public organizations, science, medicine etc., for several purposes, via different devices and with possible far-reaching consequences.

In fact, as Sellen et al. [2] highlight, people now live with technology, not just use it. In this sense, when designing people's interactions with computers and with others by using them, we have to consider new forms and dimensions of interactions that go beyond the task-oriented approach and that transcend the context of personal desktop computers.
This context brings to scene a broad set of factors that range from technical to legal and cultural ones, making the technology development an increasingly complex challenge that demands new theories, techniques, artifacts and tools for supporting their design. Cultural aspects such as values, behavior patterns, beliefs, preferences, as well as users' motivation, engagement, emotions, disabilities and the context of use are examples of concepts that have been recognized as critical to the development of technologies that meet users' needs in such new and challenging contexts.

Now, those concepts that were traditionally left on the margin of both Human-Computer Interaction (HCI) and Software Engineering approaches are moving to the centre, characterizing a new paradigm in the HCI area [3]. In this paradigm, artifacts and their contexts are mutually defining and being subject of different interpretations in a situated interaction, requiring an understanding of the establishment and multiplicity of meaning.

In this sense, researchers from academia and industry highlighted that HCI experts need to broaden the field scope and adopt new theories and methods if they are to be useful in the socio-technical environment of this scenario [2, 4, 5, 6]. Although focusing on different aspects of the same problem, they agree that we have a responsibility to ensure that the technologies we develop and deploy on society do not produce side effects that harm it; taking people culture and values in account is a basic condition for that.

However, it seems that culture and its aspects (mainly values) have become a kind of buzzword in technology design - a similar situation happened before with concepts such as usability, accessibility and, more recently, sustainability. When we talk about culture, we talk about the way of life of people, the sum of their learned behavioral patterns, attitudes, values, material things, and other aspects [1]; culture is not a single unit that can be approached as an additional non-functional requirement. Although there is a crescent appeal for works that discuss and address cultural issues in technology design, there is a lack of concrete results, 
tools, approaches and even examples of how designers can deal with such issues in an explicit way.

Indeed, particularly in the context of social software, traditional approaches based on best practices in Software Engineering (specification, design, development, testing etc.) and agile methods, created for developing Web 2.0, applications need to be rethought in order to incorporate concerns relative to cultural issues. Nonetheless, because the curricula in computer science usually neglects the education of its students with regard to social subjects, if we are to develop new solutions for furthering discussions around cultural aspects, we have to build them in a way they can support, guide and capacitate designers in their activities.

Situated in this scenario, our research projects have been directed to the development of interaction design solutions for an inclusive social web, as well as frameworks and resources to support designers in doing so. In a previous work, we proposed a culturally aware artifact to support designers in analyzing and evaluating social software, taking values and other cultural issues into account in an explicit way [7]. The artifact, named VF4SS, was applied by a group of designers to evaluate five different prototypes of systems for supporting crosscultural collaboration. The results obtained from this activity have shown that the artifact can bring effective contributions to the social software evaluation, and gave us indications that it can be applied as cultural lenses in a requirements clarification activity.

In this paper, we present and discuss the theoretical and methodological basis of our research projects and describe a case study on requirements clarification supported by the VF4SS and other artifacts from Organizational Semiotics [8]. The major goal of this case study was to make explicit identification and involvement of users' cultural aspects in the design of a computing system. We illustrate our discussion with examples from a practical context where prospective users of the solution we are envisaging were involved as active subjects. As an additional contribution, we organize and suggest activities for guiding designers to reproduce our case study in a practical context.

This paper is organized as follows: Section 2 describes the previous steps and presents the main theoretical and methodological basis used in this research. Section 3 introduces the VF4SS; Section 4 presents the case study; Section 5 suggests general activities for guiding designers in a practical context; and Section 6 presents our conclusions and directions for further research.

\section{Research Grounds}

When we talk about social software, we are not just talking about a specific set of technologies for which the focus is on people. In fact, we are talking about a kind of system that represents some of the great challenges we have to face in the digital age.

The impact of social software on people's professional, personal and social lives has been studied mainly by researchers from sociology, anthropology and communications areas. In this paper we do not enter in such discussions; rather, our focus is on the way technology can be designed in order to reflect peoples' values and culture, so that it could be naturally appropriated by them.

\subsection{Social Software}

The concept of social software as well as the particularities of its design, its elements, and the changes and challenges it brings, are being discussed in the literature and trough informal discussions on forums and blogs since the middle of 2004. A review and organization of some of those discussions are presented in [1], indicating the need of a paradigm change in the way we understand and design social software. Three main points were highlighted: two points are related to the way we design technologies, and will be discussed in the next section; the third one is related to the way we understand social software, indicating the need for approaching its elements as values - of different nature and with different relationships — instead of functional elements of software.

A set of elements in the context of social software that could be approached as values was identified in [9] through a literature review and empirical analysis of existing systems. Those elements were presented as interactive values bounded to cultural aspects (e.g., privacy, collaboration, accessibility) and some examples of how values could be promoted or inhibited by systems features were exposed. The set suggested in that paper is not exhaustive. Indeed, it is simple enough not to overload designers with a too large range of elements, while providing them with a useful list of generic elements that must be taken into account when designing social software.

In order to support designers in the evaluation of social software, the values identified in [9], complemented by an additional value (metacommunication), were embedded in an artifact to create the VF4SS (Valuation Framing for Social Software) and applied to the evaluation of five prototypes of systems for supporting cross-cultural collaboration [7]. This experiment evaluated the set of values regarding its relevance and suitability and indicated the viability of using the artifact for supporting both the evaluation and design of social software. 
In the experiment reported and discussed in [7], the VF4SS allowed designers to identify and clarify cultural aspects that resulted in new requirements for their projects; some of them could cause unexpected and undesired side effects once neglected. Although some examples had indicated it, these examples do not offer bases to reproduce such results in new situations. Therefore, it demands efforts in order to identify general activities that can guide designers to use the artifact for understanding cultural aspects favoring the requirements clarification, without limiting or narrowing them.

The identification of such activities requires attention to the two other points highlighted by Pereira et al. [1]: (i) we need to go beyond the consideration of technical issues, (ii) adopting a social perspective in social software design. Following, we describe and articulate these two points.

\subsection{Theoretical and Methodological Basis}

Traditionally, technological innovations have been produced and delivered for people to use them even without a clear perception of their utility and potential impact. Some investigations are carried out, usually by market specialists, in order to identify the overall economic viability of a given product. Cultural studies about the target audience are conducted (if any) usually to prospect selling rates. The impact that a given product (technological or not) can cause on its target audience is often neglected.

Hall [10] asserts that every innovation brings negative and/or positive impact to the environment in which it is introduced. There are people in that environment who suffer this impact, trigger others, and confer values upon such an innovation [11]. Values are defined as trans-situational goals that vary in importance and serve as guiding principles in people's lives [12] and, more generally, as something that a person, or a group of people, considers important in life [13]. In the context of technology design, Norman [14] says that people learn social skills, but machines, systems or other technological artifacts need those skills being designed into them. In this sense, as Kolkman [11] and Friedman et al. [13] argue, when designing a technology designers necessarily communicate their values, preferences and other cultural aspects. Consequently, the impact that a technology cause on its target audience may be the result of the match between the values of its users with the values of its designers.

A critical aspect regarding the values communicated through technology is that, usually, this communication is not a two-way one: unlike people with whom users can disagree and negotiate about values and their meanings they hardly can do the same with technologies [6]. Therefore, producing technologies that meet users' demands, that respect their culture and other social requirements, is possible only if designers could see the technology they are designing through the lenses of the prospective users and their socio-cultural particularities. However, as argued in [1], it requires a new Science of Design aligning system development with social practices with the end user.

Baranauskas [4] calls Socially Aware Computing "the theory, artifacts and methods we need to articulate to actually make the design socially responsible, participatory and universal as process and product". Grounded on the Organizational Semiotics theory (OS) [8] and inspired on Participatory Design [15], the author proposes a social perspective to the design of technologies, approaching it as a movement that starts in the society towards the construction of a technical system, involving representatives of the target audience as active subjects in the whole process.

To understand what this social perspective really means it is required an understanding about some basic structures of human society. Hall [10] explains that humans operate at three different levels he calls the major triad: informal, formal and technical; they are simultaneously present in everything, although one always dominate and we deal with them separately. For instance, people can learn from observing other people and imitating them (informal); they can learn from other's explicit feedback, suggestions and instructions (formal); or by books, guidelines and other materials that explain and justify things in a coherent outline form (technical). A given culture may emphasize technical learning while other may be heavily informal; on the other hand, in a same culture different levels will appear highlighted according to the context of learning: the problem-based methods used in physics are very technical while immersive approaches for cultural studies are essentially informal. Actually, we can identify all the three levels in almost any learning situation, but one will always be emphasized.

The OS theory [8] proposes a structure represented by the Semiotic Onion that explains how these levels exist in the context of organizations and information systems (see Figure 1). The informal represents the organizational culture, customs and values that are reflected as beliefs, habits and individual behavior patterns of its members. The formal corresponds to aspects that are well established and accepted, becoming social conventions, norms or laws; in this level, rules and procedures are created to replace meanings and intentions. Finally, the technical situated in the core of the onion represents aspects that are so formalized that can be technically approached and supported. 


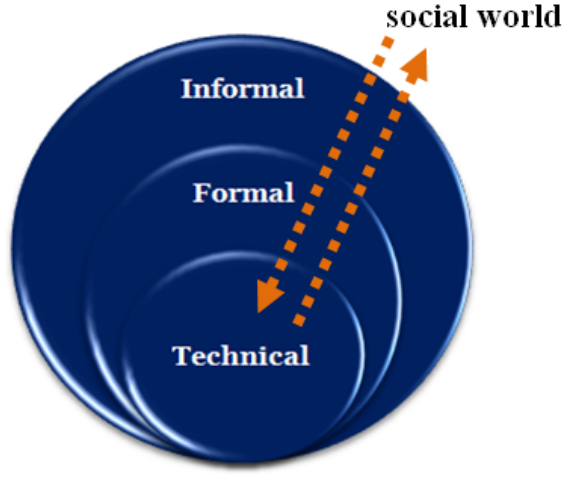

Figure 1. The Semotic Onion

In technology design, the Semiotic Onion shows that any technological artifact is situated in a formal system that, in turn, exists in the context of an informal one, suffering and causing impact on them. Consequently, as long as design processes occur centred in technical aspects regardless of the formal and informal aspects of organizations and the society, they will not be able to deal with cultural aspects in an effective way. In this sense, when Baranauskas [4] talks about a social perspective to the design of technologies, she is defending that any design process must begin from outside to inside the Semiotic Onion (see figure 1), crossing the informal and formal layers of signs towards the construction of the technical system. The technical system, in turn, will cause impact on the formal and informal layers and on society. This movement favors the identification, articulation and formalization of relevant aspects of the social world, reflecting a respect and understanding to it and favoring the production of technologies that make sense to users and are appropriated by them.

Following, we present the VF4SS and describe how it can support a requirements clarification activity grounded on the theoretical and methodological bases discussed in this Section.

\section{The VF4SS Artifact}

Understanding the potential impact of introducing an innovation requires designers to be aware of the reactions of the different groups of stakeholders who will be direct and/or indirectly affected by it. If an innovation is inserted in each group accordingly, no serious problems might occur [11]. However, sometimes there may be conflicts and designers would be able to anticipate the reactions of stakeholders only if they could see the world from these stakeholders' point of view. Although there are some techniques, such as natural observation and ethnography, which have been used in software engineering practices to assist designers in such tasks, they offer little support regarding their theoretical basis. Designers who do not have background in social sciences might have difficulties in knowing what to do or how to proceed in order to obtain relevant requirements related to people behaviors, values, conflicts, dependencies and so on. Usually, these techniques do not offer practical artifacts that can guide designers in the requirements elicitation tasks while facilitating their understanding of social issues.

The Valuation Framing (VF) is an OS's artifact [11] created on the ground of the ten building blocks of culture [10] for supporting the identification and understanding of the cultural dimensions of $a$ product. The basic principles of the VF are: all the stakeholders must be identified. These stakeholders have, in their cultural settings, a range of behavior patterns distributed into ten areas relative to the basic building blocks of culture, or Primary Message Systems (PMS) [10], e.g., Interaction, Association see the first column of Figure 2 for the complete list. The analyst's task consists of questioning, hypothesizing and predicting how the innovation could affect these stakeholders in these areas.

Hall [10] explains that each one of the 10 culture building blocks is biologically rooted, and any culture can be seen as an evolution of human behaviors and interactions mapped by a combination of them. According to him, people develop values with regard to these 10 areas. For instance, values in Association refer to the way people organize and structure themselves in society; the groups they form, the kind of relationship they develop and maintain, etc. Family is a manifestation of the aspect of association in a given culture, and its role, structure, and relative importance on society can be understood as values developed in/by that culture. In this sense, the values identified in the context of social software [9] were classified according to their suitable cultural area and, then, embedded in the VF artifact creating the VF4SS [7] (see Figure 2).

The VF4SS presents all the 10 areas (PMS) preserving the structure of the original VF and includes a column named "Value" that suggests designers possible values relative to the area. Values are also classified according to the level they are better perceived in the social software context: Informal - mostly values of personal and ethical nature, Formal - collective or social values where there is some rule or system of norms, or Technical — values that can be understood as quality attributes or special features of technology. A new column is added in the artifact for each group of stakeholder identified in the context of the solution being designed. Therefore, each area and its values can be discussed and treated in a connected but independent way for each group of stakeholders.

We have applied the VF4SS in a practical context in order to clarify requirements to the development of a social software for professionals of the Special Education field - teachers who work with students 
that have some kind of impairment. The artifact has been useful in supporting the requirement elicitation activity and in promoting discussions regarding cultural aspects. The next Section presents some examples that illustrate the artifact usage and its contributions.

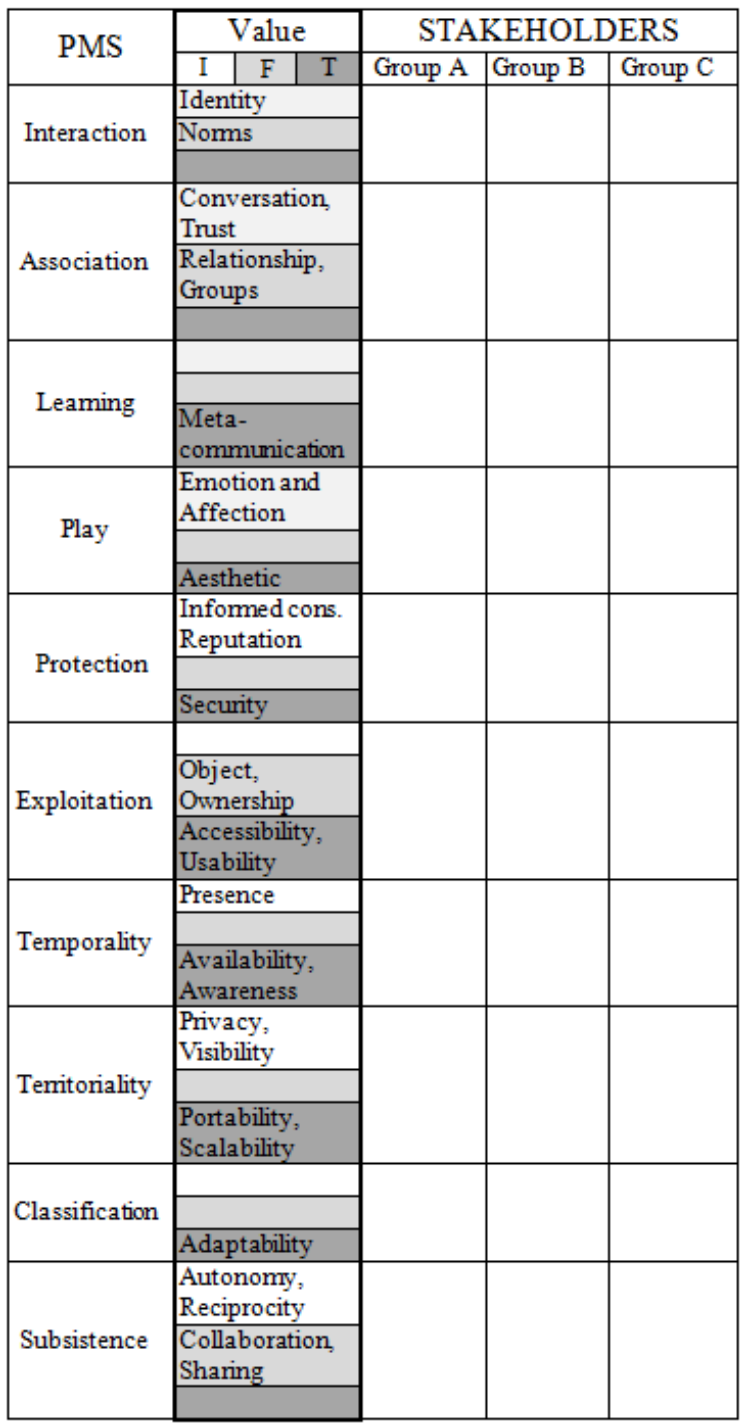

Figure 2. The VF4SS

\section{The Case Study}

In the last years, the Brazilian public policies for inclusion of disable students in regular schools created the Specialized Educational Services area (SES), in which qualified teachers accomplish activities with students in classes placed in traditional schools but equipped with specialized resources [16] — the multifunctional resources room (see Figure 3). In order to qualify professionals in this field, teachers from all over the country are being prepared for their responsibilities and activities via specialization courses within e-learning environments. However, these courses have a limited period and, after that, teachers will lose part of the support they have for accomplishing their daily activities.

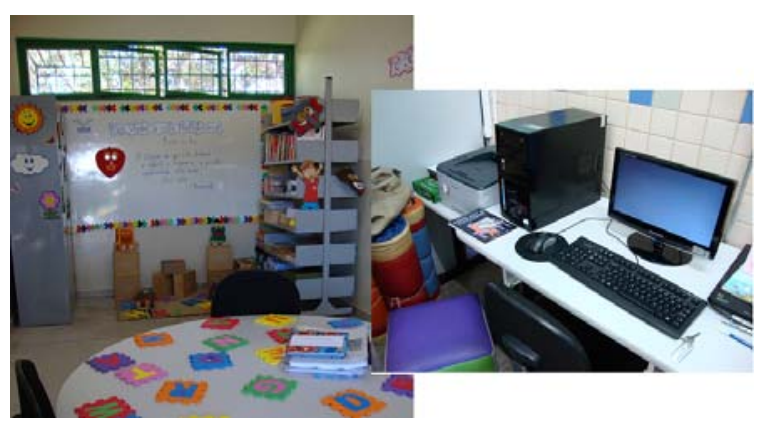

Figure 3. Multifunctional resources room [17]

In this context, we are working in a research project called TNR - Todos Nós em Rede (in English: All of Us Networked) which intends to investigate the importance and usefulness of a social network system for supporting teachers into their day by day work with real cases in a continuing education process. This network has as premises the teacher autonomy, self-regulation and the construction of knowledge about issues related to SES by the discussion of cases from their professional practices.

According to our previous discussions, designing social software is a complex task because there is a broad range of factors into play (e.g., the cultural ones) that can influence the appropriation as well as the rejection of technology. Consequently, involving prospective users through participatory activities, and understanding and dealing with their cultural aspects (e.g., values) are primary concerns in this project and are in accordance to Baranauskas' social perspective [4].

In the context of the TNR Project, we are conducting participatory activities with 28 teachers - they are considered the SES sowers - from several regions in Brazil to identify existing solutions, requirements and features for designing a social system that make sense to them. In the first stage of the participatory activities, teachers experimented four different systems for supporting them in the discussions of their cases: Yahoo! Answers [18], ACBP-Sakai [19], LeMill [20] and Vila na Rede [21] — these systems were selected in order to cover different aspects that are important to the research. Teachers suggested cases they would find interesting to discuss supported by the selected 


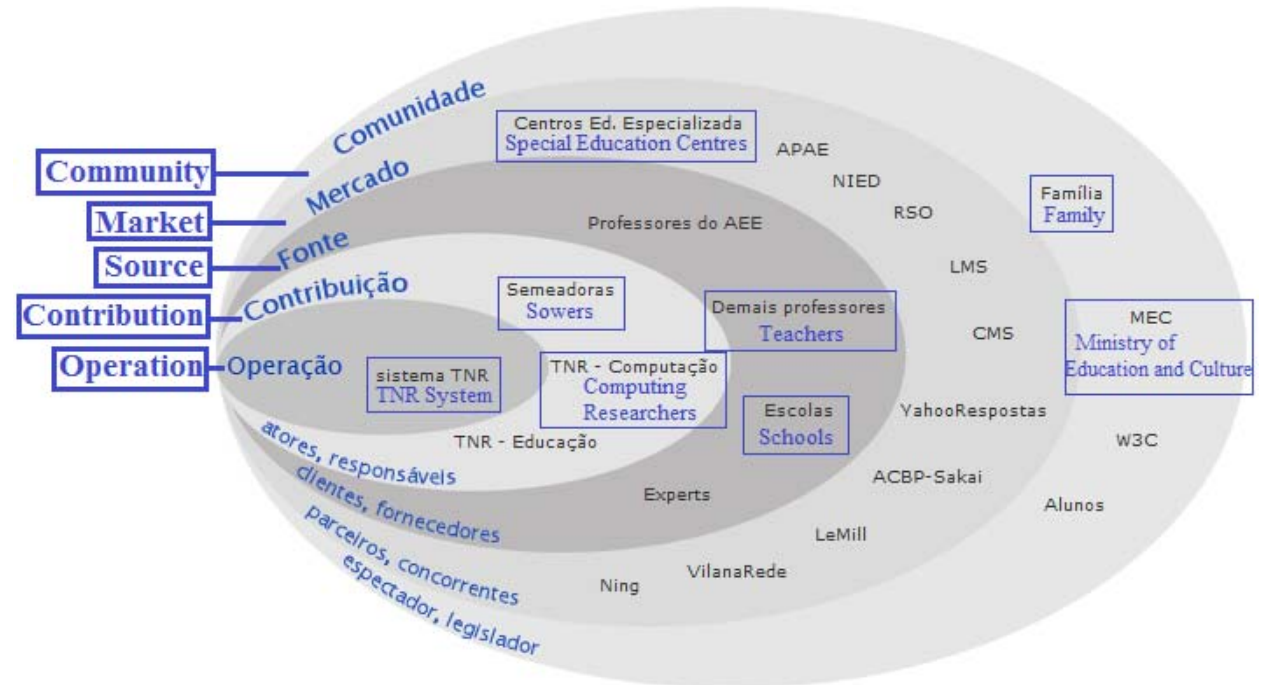

Figure 4. The Stakeholders Identification Diagram

systems and the research team chose four of them (one per system), creating four different scenarios. The main goal in each scenario, however, was not solving the case itself but evaluating how useful these systems could be in supporting online construction of a case solution in teachers' real practices. Following, we describe a clarification activity conducted on the data produced in the first scenario.

The system used in this first scenario was the Yahoo! Answer [18]. This system was chosen because it allows users to interact in a questionanswer scheme. For their interactions, we posted the description of the first case as a question in the system and asked them to come to a solution for it. After about a month using the system, teachers gave us their feedback by: i) their interaction through the system in order to solve the proposed case; ii) answering an evaluation questionnaire, pointing out the resources they liked, disliked, missed etc., and their opinion regarding the benefits and limitations the system brought to their discussions; and iii) a semi-structured interview with the researchers team. Then, we analyzed these data through the VF4SS's lenses in order to know users and their contexts (abilities, preferences, limitations, values etc.), drawing attention to the requirements elicitation for the system we are designing. The procedure we carried out was as follows:

First, we identified some groups of stakeholders involved in the solution we are designing, for instance: the Sowers, Computing researchers, Education researchers, Teachers, Students, Family, Schools, MEC (Ministry of Education and Culture) etc. The Stakeholders Identification (SID) diagram [8] from OS was used in this activity (see Figure 4 - translations were made by the authors): it distributes the groups of stakeholders in different categories ranging from the actors directly involved in the project (e.g., direct users such as the Sowers) to the community who may not use the system but can be affected by it (e.g., students' family). We selected the four most representative ones in the context of our Project, covering informal, formal and technical aspects: Sowers (informal), MEC (Formal), Education Researchers (Formal), and Computing researchers (Technical), inserting them as a new column into the VF4SS.

Second, we discussed how the different groups of stakeholders would understand each area and the values suggested in the artifact. For instance, regarding the aspect of "Association" sowers expect a system for supporting the discussion of their cases and for interacting with other teachers who have similar interests and/or difficulties. In the evaluation of the Yahoo! Answers, they pointed out that it does not support the value of conversation in an effective way; using a sower's words: “(...) the system did not allow an effective discussion of the case. I felt frustrated when trying to complement my answers" and "it is not dynamic; I felt lack of dialogue with other participants". This kind of complaint indicates that the value of "conversation" is very important to them for an effective interaction, having to be as flexible as possible. This feedback also gave us a cultural clue: it seems that teachers are not seeking for closed answers to their problems; instead, they expect a solution built from their discussions and ideas, and that will be suitable to their problems and context.

Third, we highlighted the values identified in sowers' answers, evaluations or interviews, discussed their scope and possible means for technically supporting them. For instance, sowers suggested they would want to complement their 
answer because while other users post comments in a case, they could have new comments and ideas to show. They reported that: "when I want to complement my answer, after a few days, I am not able to do it"; "I read everything, but I could not answer other comments. I like chats, where I can interact with others and know the opinion of my colleagues"; and "Once answered, I was not able to answer again in order to complement my answer or to post a question to a colleague. I could not reply comments or develop a discussion". This kind of feedback shows us that the discussion is not a linear one, but iterative and collectively built. In this sense, a scheme similar to a forum might be more appropriate than a question-answer one.

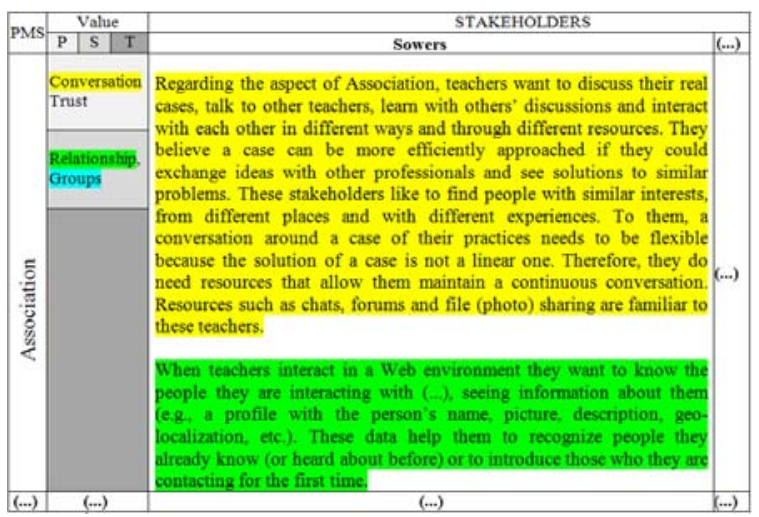

Figure 5. VF4SS filled in the aspect of association

Fourth, we analyzed the highlighted values looking for possible conflicts between the different groups of stakeholders. For instance, we identified a possible threat to the value of privacy in the perspective of students caused by the value of conversation in the sowers' perspective. When asked about their concern with privacy issues sowers answered: "I have no problem with that [on privacy]"; "I am not worried about that because my answers are based on my practices" and "(...) in any social network we stay very exposed; there is no other way. I think it is important to share my thoughts (...)”. Some authors talk about privacy in social networks arguing that privacy is not well understood and/or considered as important by beginners while it is considered critical by expert users; Satchell and Dourish [22] consider that "privacy is a concern only for the concerned". However, because the cases being discussed are real cases, the concern with privacy here cannot be restricted to teachers. The identification of students and their personal information, for instance, may have deep and far-reaching consequences. Nevertheless, this type of concern was mentioned as important by only one sower during the whole activity. In this sense, the system we are designing has to let teachers aware of the impact the information they are producing can have, instructing them regarding privacy concerns - it corresponds to a value we call informed consent.

Fifth, we discussed how values, such as the informed consent, could be technically supported in the system. For instance, tips and advises regarding privacy issues could be displayed in the system interface, and mechanisms for controlling the visibility of the information produced by the users could be developed. For instance, when users create a new announcement at the Vila na Rede system [21], they have to configure whether the content will be visible only for registered users or for everyone who access the system; it also offers a feature that explains to users, using video, audio or pictures, the purpose of that configuration.

Finally, we could elicit requirements associated to values in the context of the solution we are seeking to design. These requirements can be formalized, for example, by defining norms [8]. A formalized norm for advising users regarding privacy issues when they are discussing a case could be: "whenever a new case is posted, if it is publicly available then the user is obliged to confirm his/her awareness about the risks of exposing personal information or any data that may affect his/her privacy or the privacy of other people”. This norm can also be treated as a functional requirement.

\subsection{Discussion}

The VF4SS enables designers looking at the system being projected through the lenses of different stakeholders who would be affected by it in different ways. Besides, it also supports designers in identifying the values involved in their system and the way these values can be/are being technically supported. In fact, the values suggested in the artifact act as a starting point from which designers can elaborate further discussions and investigations. Hence, the suggested values are important not only to support designers to carry out the analysis of their projects, but to understand each PMS, discover new values and learn how to use the artifact itself.

In a requirement elicitation activity, one of the main contributions of the VF4SS is its capability of generating fruitful discussions among designers, allowing them to exercise a critical thinking with respect to the whole impact of the solutions they are designing. If applied in the early stages of software development, this artifact can effectively contribute to the creation of products compatible with the values of people they are intended for instead of the values of their designers, benefiting the system deployment and stakeholders' appropriation.

Regarding the artifact application, it was possible to identify some interesting points: 1 . No matter the design process or the software development model adopted in a project, the artifact can provide 
theoretical basis and promote further discussions in terms of values and culture. 2. The task of using the artifact is not linear; designers will move back and forth identifying new cultural aspects, discussing new values, revising requirements and so on. 3 . Although the VF4SS brings the lenses of culture and values to the interaction design of systems, contributing to an understanding of the system from different points of view, it does not substitutes the figure of the user. Indeed, the artifact serves as a guide regarding cultural issues, and it is better applied in practical settings where representatives from the target audience are involved.

Besides identifying and clarifying requirements in the project context, the activity provided some indications for the next steps of the system design. Because they seem to be valid for the project of any technological artifact, they are presented in a generalized way:

First, it is necessary to take into account the views and interests of different groups of stakeholder. As previously argued, different stakeholders understand, value, cause and suffer the impact of an innovation in different ways. They also may influence/be influenced by other groups of stakeholders.

Second, discussing cultural aspects provide rich information about the target audience and the environment in which the solution being designed will be inserted. As illustrated by the Semiotic Onion, considering such aspects corresponds to consider part of the informal and formal systems that contribute to a fully comprehension about the problem domain.

Third, as Schwartz demonstrates in his circular model [12], values have an interactive nature. When a certain value is promoted or inhibited, it can affect other values, promoting or inhibiting them. Sometimes, a value can be promoted only by promoting other values; on the other hand, there are cases that a value has to be neglected in favor of others. Being aware of such interactive nature among the values themselves contributes to avoid pitfalls in the attempt of promotinglinhibiting a certain value.

Forth, it is possible to move from informal discussions regarding cultural aspects to formal requirements that can be technically approached in a way analogous to Baranauskas' proposal [4]. This movement favors the clarification and elicitation of requirements that carry the concern for culture with them.

\section{A Practical Guide for the VF4SS}

Based on our examples and discussions, in this Section we suggest some general activities for guiding designers to apply the VF4SS in the practical context of an industrial setting as well as in a research environment.
The Figure 6 illustrates these activities indicating the artifacts that support them. The light blue rectangles represent general activities, while the rounded orange rectangles represent their outcomes. Solid arrows indicate an input or a result of an activity, while dashed arrows indicate that the activity can generate a re-input to another. The requirements clarification starts with the identification of the groups of stakeholders, is iterated and finishes when designers conclude they have clarified the relevant areas and their values for the groups of stakeholders selected, identifying requirements to the project.

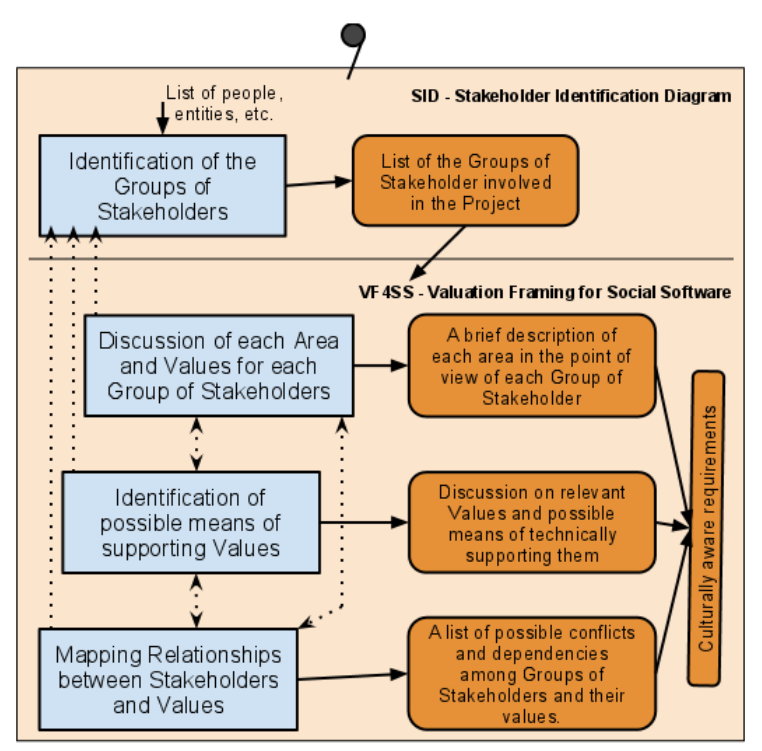

Figure 6. General activities for guiding designers

Identify the groups of stakeholder direct or indirectly involved/interested in the system being developed. The SID artifact [8] can support this task. As a result, a new column for each group of stakeholders is included in the VF4SS. For practical reasons, we suggest selecting the most representative ones to the context of the project being developed. In order to keep a wide perception of the problem domain and the different stakeholders, consider selecting at least one group from each SID's layer.

The income of this activity can be the SID to be filled and a list of the people who are directly interested in the system development (e.g., teachers, researchers and students); the outcome is the SID filled with the groups of stakeholders direct and/or indirectly involved and/or interested in the system being projected.

Make questions, hypothesize and try to predict how the system can affect the groups of stakeholders regarding each area, and the way these groups understand, see and give importance to them - the suggested values can serve as a starting point for this kind of reasoning. For instance, how do teachers see the system regarding the aspect of Learning? Is the 
system an opportunity for new learning by discussing their cases? Is it complex to learn how to operate the system? This activity allows designers to see the system being projected through the lenses of the different stakeholders groups and to understand how the system development may affect these stakeholders. If new values are identified in this activity, they can be added into the VF4SS.

Considering the values suggested or inserted in the VF4SS during the requirements clarification, discuss the scope and relevance of each value for each group of stakeholders and identify possible means of supporting these values in the system design. For instance, regarding Learning it could be identified that teachers want to discuss their cases in the system in order to improve their knowledge and experience. However, they do not know whether the system controls the visibility of information; so, they are afraid that publicly exposing their doubts may negatively affect their reputation in the work environment as well as in their personal lives. In this example, we can see both a concern regarding the possible impacts of i) using the system to ask for help and ii) operating the system itself. The first concern puts the values of privacy, reputation and trust in evidence and highlights the need for creating mechanisms that allow users to manage the visibility of the information they produce. The second one draws attention to the values of autonomy, usability, accessibility, meta-communication and informed consent, indicating that the system must be easy to learn, understand and operate, instructing users and letting them aware of the possible results of their actions. This activity favors the understanding of the way the values involved in the context of the system being projected are manifested, the relationships among them and the way these values can be technically supported.

Analyze and map the possible relationships between the different stakeholder groups regarding the analyzed values and specify means for supporting them (e.g., technical features, project constraints). Sometimes there are dependencies and even conflicts between different groups of stakeholders that must be taken into account in the system design. These relationships can be explicit, e.g., if the system is moderated then the teacher depends on moderators' approval to post a new case; or they can be implicit, e.g., the school director may not approve the teacher using the system because the exposition of information regarding school's cases, practices, rules and structure can be seen as a possible threat to him. The outcome of this activity is a mapping of the relationships between stakeholders' values that must be considered in the project and possible means of dealing with them. In some cases, as in the conflict between the teacher and the school director, a technical feature is not enough to solve a conflict or support a dependency, and perhaps there will be few (if any) possible ways of dealing with that. However, designers need to be aware of these conflicts and dependencies in order to reduce them (e.g., providing clear and effective privacy control resources) or, at least, to avoid making them harder.

When designers discuss a way of supporting a value or dealing with a conflict, they are also discussing about functionalities, constraints, quality aspects etc. that can be understood as requirements to the project. Hence, the discussions generated in these activities favor the identification of requirements contributing to the understanding of the environment in which the solution being projected will be introduced; it, in turn, favors the development of a solution that reflects a respect to the culture and values of its intended users, instead of the cultural aspects of its designers. As we mentioned before, the results obtained from the artifact usage can be even more promising if representatives from the target audience are involved in the process.

\section{Conclusion}

In this paper we approached the design of social software as a challenging task where values, emotion, motivation, and several other cultural aspects play a central role. Consequently, new theories, approaches, artifacts and tools for supporting designers when projecting systems placed in this scenario are needed. Within this context, we presented the VF4SS as an artifact for supporting designers in a requirements clarification activity. Then, we presented some examples, derived from a practical context, of how the artifact can support designers from informal discussions to requirements clarification, and suggested general activities for guiding them in the artifact usage.

The artifact as well as the general activities and the discussions we presented in this paper are grounded on Organizational Semiotics [8], the building blocks of culture [10], the Socially Aware Computing approach [4] and Schwartz's values model [12]. In this sense, we tried to present our research articulating theory and practice, exposing arguments and examples, so that the reader who is not familiar with such theoretical-methodological basis can understand it.

As future work, we are projecting a case tool for supporting the use of the VF4SS. The development of such tools and conceptual framework are part of a project named EcoWeb [23] that aims to empower professionals and researchers involved with the design and development of social systems to produce solutions that effectively meet users' requirements, taking into account their differences, needs, preferences, limitations, values and other cultural aspects. 


\section{Acknowledgements}

This research is partially funded by FAPESP (grants \#2009/11888-7 and \#2010/11004-9), CNPq via the EcoWeb Project (\#560044/2010-0 and \#383536/2011-1) and Proesp/CAPES via the TNR Project (\#23038.01457/2009-11). The authors specially thank the Teachers that participate in the case study used in this paper and the anonymous reviewers that contributed through their comments and evaluation.

The present paper is a reviewed and extended version of a previous work published at i-Society 2011 - International Conference for Information Society, entitled "Interaction Design of Social Software: Clarifying requirements through a culturally aware artifact”. The authors thank IJDS editors for the invitation.

\section{References}

[1] Pereira, R., Baranauskas, M. C. C. and Silva, S. R. P. (2010) "A discussion on social software: concept, building blocks and challenges”, IJI - International Journal for Infonomics, Vol. 3 (4), pp. 382-391.

[2] Sellen, A., Rogers, Y., Harper, R. and Rodden, T. (2009) "Reflecting human values in the digital age", Communications of the ACM, Vol. 52, pp. 58-66.

[3] Harrison, S., Tatar, D. and Sengers, P. (2007) "The three paradigms of HCI”, in Proceedings of the ComputerHuman Interaction Conference (AltCHI'07), pp.1-21.

[4] Baranauskas, M. C. C. (2009) "Socially aware computing", in Proceedings of the VI International Conference on Engineering and Computer Education, Buenos Aires, pp. 1-4.

[5] Hendler, J., Shadbolt, N., Hall, W., Berners-Lee, T. and Weitzner, D. (2008) "Web science: an interdisciplinary approach to understanding the web", Communications of the ACM, Vol. 51, pp. 60-69.

[6] Friedman, B. (1996) "Value-Sensitive Design". Interactions. p.16-23.

[7] Pereira, R. and Baranauskas, M. C. C. (2011) "Valuation Framing for Social Software: A Culturally Aware Artifact". in Proceedings of the 13th International Conference on Enterprise Information Systems (ICEIS), Beijing, pp. 135-144.

[8] Liu, K. (2000) "Semiotics in information systems engineering”, Cambridge, University Press.

[9] Pereira, R., Baranauskas, M. C. C. and Silva, S. R. P. (2010) "Softwares Sociais: uma visão orientada a valores". In Proceedings of IX Brazilian Symposium on Human Factors in Computer Systems, ACM, pp. 149-158.

[10] Hall, E. T. (1959) “The silent language”, Anchoor Books.
[11] Kolkman, M. (1993) "Problem articulation methodology”, University of Twente, Enschede.

[12] Schwartz, S. H. (2005) "Basic human values: their content and structure across countries", Values and Behaviors in Organizations, Vozes.

[13] Friedman, B. Kahn, P. H. and Borning, A. (2006) "Value sensitive design and information systems", HumanComputer Interaction and Management Information Systems: Foundations, Armonk, pp. 348-372.

[14] Norman, D. (2008) “Sociable design”, http://www.jnd .org/dn.mss/sociable_design_intr.html, (30 July 2011).

[15] Schuler, D. and Namioka, A. (1993) "Participatory design: principles and practices”, Hillsdale, Lawrence Erlbaum Associates.

[16] MEC. (2009) "Brazilian national policy on special education in the perspective of inclusive education" (Portuguese),http://portal.mec.gov.br/arquivos/pdf/politica educespecial.pdf, (24 April 2011).

[17] Barbosa, M. C. (2010) “A Importância de uma Rede Social para o Aperfeiçoamento Continuado dos Profissionais do Atendimento Educacional Especializado”. Technical Report for the Brazilian Culture and Education Ministry.

[18] Yahoo! Answers, http://answers.yahoo.com, (30 July 2011).

[19] ACBP-Sakai, http://styx.nied.unicamp.br:8082, (30 July 2011).

[20] LeMill, http://lemill.net, (30 July 2011).

[21] Vila na Rede, http://www.vilanarede.org.br/, (30 July 2011).

[22] Satchell, C. and Dourish, P. (2009) "Beyond the User: Use and Non-Use in HCI". in Proceedings of OZCHI'2009. ACM.

[23] EcoWeb Project, http://www.nied.unicamp.br/ecoweb (30 July 2011). 